\title{
Radiative accelerations in stars: The effects of magnetic polarisation revisited
}

\author{
G. Alecian ${ }^{1}$ and M. J. Stift ${ }^{2}$ \\ ${ }^{1}$ LUTH (Observatoire de Paris - CNRS), Observatoire de Meudon, 92195 Meudon Cedex, France \\ 2 Institut für Astronomie (IfA), Universität Wien, Türkenschanzstrasse 17, 1180 Wien, Austria \\ e-mail: stift@astro.univie.ac.at
}

Received 6 October 2003 / Accepted 17 November 2003

\begin{abstract}
We present a revision of the results obtained by Alecian \& Stift (2002) on the amplification, by Zeeman splitting in strong stellar magnetic fields, of radiative accelerations of chemical elements. These results had been obtained for blended spectra and were based on the Zeeman Feautrier method as presented by Rees et al. (1989) which however requires perfect symmetry of the line profiles. The use of this method in an inappropriate context led to the incorrect identification of those line absorption terms which change sign for the incoming radiation. The question of magnetic amplification of radiative accelerations had to be revisited. Following the formulation of an alternative Zeeman Feautrier scheme which remains valid for blends (albeit only when macroscopic velocity fields are excluded), the resulting radiative accelerations are now less amplified than what had been found in Alecian \& Stift (2002). In a $12000 \mathrm{~K}, \log g=4.0$ Kurucz atmosphere with solar abundances, amplifications at a field strength of $4 \mathrm{~T}$ peak at about $0.4 \mathrm{dex}$, and there is very little dependence on the field inclination. Depending on the Zeeman pattern, individual lines may exhibit amplifications of more than 1.1 dex. Blending is found to greatly affect radiative accelerations but not amplifications; only in exceptional cases such as for Ag can strong blending lead to an inversion of the amplification, i.e. accelerations actually decrease with increasing field strength. Finally magneto-optical effects continue to be non-negligible, horizontal accelerations remain small.
\end{abstract}

Key words. diffusion - stars: abundances - stars : chemically peculiar - stars: magnetic fields - polarization

\section{Introduction}

The modelling of abundance inhomogeneities in the atmospheres of magnetic Ap stars is one of the interesting challenges in contemporary stellar astrophysics. The abundance peculiarities observed in the photosphere of these stars are supposedly due to microscopic diffusion which, in the presence of magnetic fields, leads to stratification of the chemical elements. Since very strong magnetic fields can be found in the atmospheres of these stars, a major aspect in the modelling of the horizontal and the vertical distribution of the various elements is the evaluation of the effect of Zeeman splitting on the radiative accelerations. A pioneering work, based both on analytical approximations to the polarised radiative transfer problem and on numerical solutions, was carried out by Babel \& Michaud (1991) (henceforth BM). More recently, we have investigated the same problem in detail by numerical means (Alecian \& Stift 2002, hereafter Paper I). The exhaustive computations were carried out for 329 ions, using the VALD atomic database (Piskunov et al. 1995), solving numerically the polarised

Send offprint requests to: G. Alecian,

e-mail: georges.alecian@obspm. fr radiative transfer equation by means of the Zeeman Feautrier method (Auer et al. 1977; Rees et al. 1989) including magnetooptical effects, and providing for the correct treatment of line blending. In Paper I we presented results that show that in a few metals, magnetic amplifications can become very large for field strengths in excess of $1 \mathrm{~T}$. In addition, we found that these amplifications strongly depend on the field angle with respect to the vertical.

However, despite the impressive number of tests passed by the CARAT code, we continued the verifications, striving for a better understanding of the apparent lack of correlation between the magnetic amplification of radiative accelerations and the increase in equivalent width (the so-called magnetic intensification). More complicated anomalous Zeeman patterns normally lead to increased intensification (see Stift \& Leone 2003) but magnetic amplifications as determined in Paper I proved to behave the opposite way (e.g. in Fig. 2 of Paper I). Finally we were led to examine more closely the Zeeman Feautrier method and Eq. (12) of Paper I which gives the acceleration vector. It became clear to us that the Zeeman Feautrier method as presented by Rees et al. 1989) is not applicable to blends since it is based on the assumption of perfect symmetry of the 
line profiles (although this is nowhere explicitly mentioned). In this context it also emerged that we had not properly identified those line absorption terms which change sign for the incoming radiation. All this means that the accelerations determined in Paper I have to be revised.

In the present paper, we concentrate on the changes with respect to Paper I, and present some new results. Details on the CARAT code and its numerics can be found in Paper I; apart from one crucial line of code in the polarised formal solver, nothing was changed. We first develop a new formulation of the Zeeman Feautrier method that allows the correct treatment of blends in Sect. 2, and in Sect. 3 we reformulate detailed expressions for the radiative accelerations in a magnetic atmosphere. After the presentation of the new results, we have a close look at heavy blending, at the dependence of magnetic amplification on effective temperature, at amplification as a function of Zeeman pattern, and at the importance of magneto-optical effects.

\section{Polarised radiative transfer: The Zeeman Feautrier method}

The results presented in Paper I are based on the Zeeman Feautrier method discussed in substantial detail by Rees et al. (1989). Radiative accelerations (which depend on the net Stokes $f u x$ ) require a formal solver for the polarised radiative transfer equation that yields accurate fluxes from the highest layers down to the bottom of the atmosphere and that is why we chose the Feautrier scheme which automatically recovers the diffusion approximation at great depths (Mihalas 1978) and is renowned for its stability. Line blending at short wavelengths can be quite heavy in most CP stars and we took it for granted that the Zeeman Feautrier solver, in analogy to the unpolarised Feautrier solver (Feautrier 1964), can be applied to polarised blends, although only in static atmospheres. Indeed, neither Rees \& Murphy (1987) nor Rees et al. (1989) explicitly mention any restrictions as to the line opacity profile, but in fact they made the same assumptions as Auer et al. (1977), viz. that absorption and emission profiles be symmetric in frequency.

The choice by Auer et al. (1977) was dictated by the desire to be able to treat macroscopic velocity fields. The same is true for Rees et al. (1989) who included magneto-optical terms into their Zeeman Feautrier solution. Restricting themselves explicitly or implicitly to symmetric profiles, both therefore chose to define the outgoing Stokes vector $\boldsymbol{I}(+v,+\boldsymbol{n})$ on one side of the spectral line, the incoming Stokes vector $I(-v,-\boldsymbol{n})$ on the other side. This scheme turns out unsuitable for blends. In contrast, BM define a different incoming Stokes vector $I(+v,-n)$ on the same side as the outgoing Stokes vector $\boldsymbol{I}(+v,+\boldsymbol{n})$ which enables the treatment of blends but only when there are no macroscopic velocity fields. Their statement that they are following Rees \& Murphy (1987) is thus misleading. We note that the approach by BM does not include magneto-optical terms and that it uses a special reference frame in which Stokes $U$ is always zero.

With some surprise we note that more than a quarter of a century after Auer et al. (1977) there is still no polarised Zeeman Feautrier formal solver available that is applicable to blends in static atmospheres and that correctly takes into account magneto-optical effects. There can hardly be any doubt that such a formal solver must exist, but it has yet to be established.

\subsection{A new formulation of the Zeeman Feautrier method}

In the following we shall stick largely to the notation used by Rees et al. (1989) in order to facilitate comparison, and we follow the same path as Rees \& Murphy (1987) in developing our equations. Since we stay resolutely on one side of the line, $\boldsymbol{I}(+v,+\boldsymbol{n})$ simply becomes $\boldsymbol{I}(+\boldsymbol{n})$ whereas $\boldsymbol{I}(+v,-\boldsymbol{n})=\boldsymbol{I}(-\boldsymbol{n})$ replaces $\boldsymbol{I}(-v,-\boldsymbol{n})$. With the Stokes vector $\boldsymbol{I}=\{I, Q, U, V\}^{\dagger}-$ let us recall that $I$ represents the intensity, $Q$ and $U$ characterise the linear polarisation, and $V$ the circular polarisation the vector transfer equation for polarised light can be written as

$\frac{\mathrm{d}}{\mathrm{d} s} \boldsymbol{I}=-\boldsymbol{K} \boldsymbol{I}+\boldsymbol{j}$

where $s$ is the spatial coordinate along the ray path $(z$ in the notation of Rees et al. 1989). The absorption matrix (line plus continuum) $\boldsymbol{K}$, the total emission vector $\boldsymbol{j}$, and the Stokes vector $\boldsymbol{I}$ are all functions of frequency $v$.

$\boldsymbol{K}=\kappa_{\mathrm{c}} \mathbf{1}+\kappa_{\circ} \boldsymbol{\Phi}$

$\boldsymbol{j}=\left(\kappa_{\mathrm{c}} B_{v} \mathbf{1}+\kappa_{\circ} S_{\mathrm{L}} \boldsymbol{\Phi}\right) \boldsymbol{I}_{\circ}$.

Here $\kappa_{\mathrm{c}}$ stands for the continuum opacity, $\kappa_{\circ}$ denotes the line centre opacity for zero damping and zero magnetic field. $B_{v}(T)$ is the Planck function, $S_{\mathrm{L}}$ the scalar line source function, $\mathbf{1}$ the unit $4 \times 4$ matrix, and $\boldsymbol{I}_{\circ}=\{1,0,0,0\}^{\dagger}$. The line absorption matrix

$\boldsymbol{\Phi}=\left(\begin{array}{rrrr}\phi_{I} & \phi_{Q} & \phi_{U} & \phi_{V} \\ \phi_{Q} & \phi_{I} & \phi_{V}^{\prime} & -\phi_{U}^{\prime} \\ \phi_{U} & -\phi_{V}^{\prime} & \phi_{I} & \phi_{Q}^{\prime} \\ \phi_{V} & \phi_{U}^{\prime} & -\phi_{Q}^{\prime} & \phi_{I}\end{array}\right)$

depends on strength, direction $\gamma$ and azimuth $\chi$ of the magnetic field vector $\boldsymbol{B}$. The line absorption terms become

$$
\begin{aligned}
\phi_{I} & =\frac{1}{4}\left(2 \phi_{\mathrm{p}} \sin ^{2} \gamma+\left(\phi_{\mathrm{r}}+\phi_{\mathrm{b}}\right)\left(1+\cos ^{2} \gamma\right)\right) \\
\phi_{Q} & =\frac{1}{4}\left(2 \phi_{\mathrm{p}}-\left(\phi_{\mathrm{r}}+\phi_{\mathrm{b}}\right)\right) \sin ^{2} \gamma \cos 2 \chi \\
\phi_{U} & =\frac{1}{4}\left(2 \phi_{\mathrm{p}}-\left(\phi_{\mathrm{r}}+\phi_{\mathrm{b}}\right)\right) \sin ^{2} \gamma \sin 2 \chi \\
\phi_{V} & =\frac{1}{2}\left(\phi_{\mathrm{r}}-\phi_{\mathrm{b}}\right) \cos \gamma
\end{aligned}
$$

and the Faraday terms

$$
\begin{aligned}
\phi_{Q}^{\prime} & =\frac{1}{4}\left(2 \phi_{\mathrm{p}}^{\prime}-\left(\phi_{\mathrm{r}}^{\prime}+\phi_{\mathrm{b}}^{\prime}\right)\right) \sin ^{2} \gamma \cos 2 \chi \\
\phi_{U}^{\prime} & =\frac{1}{4}\left(2 \phi_{\mathrm{p}}^{\prime}-\left(\phi_{\mathrm{r}}^{\prime}+\phi_{\mathrm{b}}^{\prime}\right)\right) \sin ^{2} \gamma \sin 2 \chi \\
\phi_{V}^{\prime} & =\frac{1}{2}\left(\phi_{\mathrm{r}}^{\prime}-\phi_{\mathrm{b}}^{\prime}\right) \cos \gamma .
\end{aligned}
$$

The line absorption profiles for the $\pi$ and for the blue or red shifted $\sigma$ components - the shifts are directly proportional to the magnetic field strength - are denoted by $\phi_{\mathrm{p}, \mathrm{b}, \mathrm{r}}$ respectively, and $\phi_{\mathrm{p}, \mathrm{b}, \mathrm{r}}^{\prime}$ are the corresponding anomalous dispersion profiles. 
Feautrier (1964) has shown that the first-order radiative transfer equation can be transformed - after splitting the specific intensity into 2 parts, viz. the outgoing intensity $I(+\boldsymbol{n})$ and the incoming intensity $\boldsymbol{I}(\boldsymbol{- n})-$ to a second-order differential equation with a number of desirable properties, including second to fourth-order accuracy, excellent numerical stability and the automatic recovery of the diffusion approximation at great depths. His scheme suffers one restriction: in the presence of macroscopic velocity fields the line absorption profile has to be symmetric, blends can be treated correctly only in static atmospheres.

Auer et al. (1977) were the first to extend Feautrier's approach to polarised radiative transfer. One has to be careful with the signs of the line absorption and the Faraday terms, since both the inclination $\gamma$ and the azimuth $\chi$ under which the magnetic field vector $\boldsymbol{B}$ is seen, change between the outgoing pencil of radiation $I(+\boldsymbol{n})$ and the incoming pencil $\boldsymbol{I}(-\boldsymbol{n})$. The local geometry of the magnetic stellar atmosphere is sketched in Fig. 1. The right-handed reference frame $x y z$ is defined such that a rotation about the $x$-axis of the magnetic field vector $\boldsymbol{B}=\{0,0, B\}^{\dagger}$ by the angle $-\beta$ makes the resulting $z^{\prime}$-axis coincide with the vertical direction in the stellar atmosphere. The rotation about the $z^{\prime}$-axis by the angle $\psi$ and the subsequent rotation about the resulting new $x^{\prime \prime}$-axis by the angle $\theta$ yield $\boldsymbol{B}^{\prime \prime \prime}=\left\{B_{x}^{\prime \prime \prime}, B_{y}^{\prime \prime \prime}, B_{z}^{\prime \prime \prime}\right\}=S_{\theta} S_{\psi} S_{\beta} \boldsymbol{B}$ the components of the magnetic field vector in the system of the outgoing pencil of radiation. We have the following expressions

$B_{x}^{\prime \prime \prime}=-B(\sin \beta \sin \psi)$

$B_{y}^{\prime \prime \prime}=-B(\cos \theta \sin \beta \cos \psi-\sin \theta \cos \beta)$

$B_{z}^{\prime \prime \prime}=+B(\sin \theta \sin \beta \cos \psi+\cos \theta \cos \beta)$

where

$S_{\beta}=\left(\begin{array}{rrr}1 & 0 & 0 \\ 0 & \cos \beta & -\sin \beta \\ 0 & \sin \beta & \cos \beta\end{array}\right)$

$S_{\psi}=\left(\begin{array}{rrr}\cos \psi & \sin \psi & 0 \\ -\sin \psi & \cos \psi & 0 \\ 0 & 0 & 1\end{array}\right)$,

$S_{\theta}=\left(\begin{array}{rrr}1 & 0 & 0 \\ 0 & \cos \theta & \sin \theta \\ 0 & -\sin \theta & \cos \theta\end{array}\right)$.

Whence the angles $\gamma$ and $\chi$ for the outgoing pencil of radiation as required in Eqs. (5)-(11) become

$\cos \gamma=\sin \theta \sin \beta \cos \psi+\cos \theta \cos \beta$
$\tan \chi=\frac{\cos \theta \sin \beta \cos \psi-\sin \theta \cos \beta}{\sin \beta \sin \psi}$.

The incoming pencil of radiation requires a third rotation about the $x^{\prime \prime}$-axis by the angle $\theta+\pi$. With

$S_{\theta+\pi}=\left(\begin{array}{rrr}1 & 0 & 0 \\ 0 & \cos (\theta+\pi) & \sin (\theta+\pi) \\ 0 & -\sin (\theta+\pi) & \cos (\theta+\pi)\end{array}\right)$

the components of the magnetic field vector in the system of the incoming pencil of radiation now become $\boldsymbol{B}_{-}^{\prime \prime \prime}=S_{\theta+\pi} S_{\psi} S_{\beta} B$
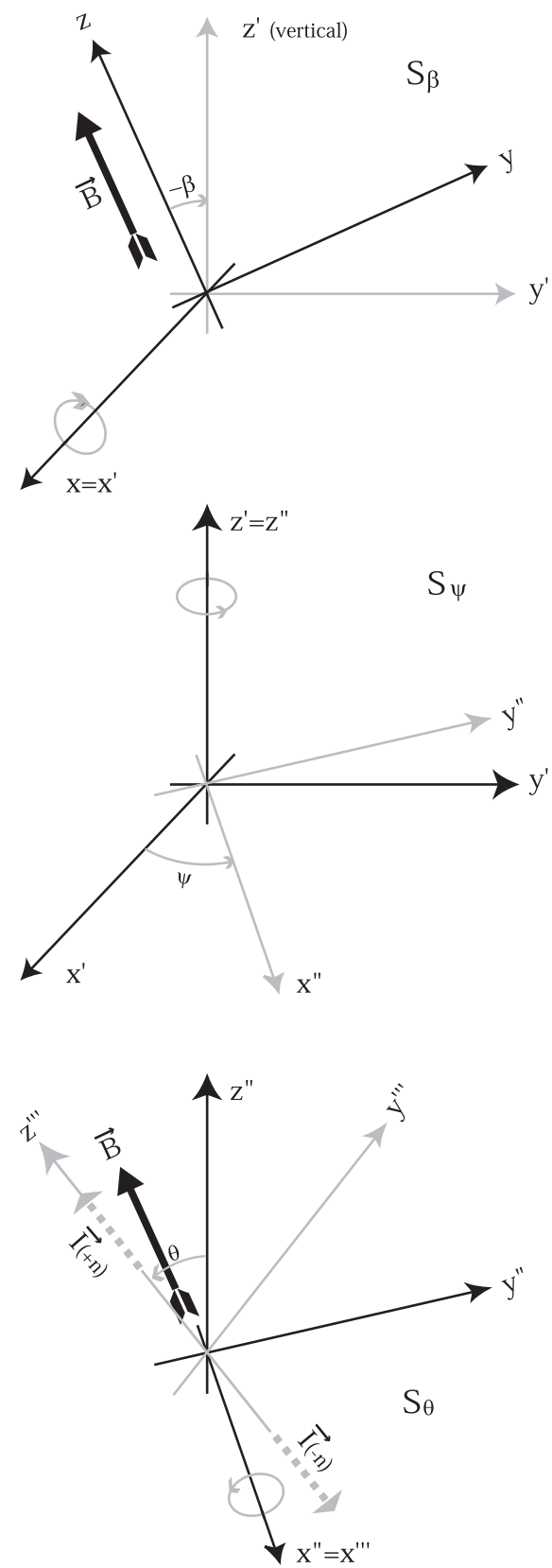

Fig. 1. The 3 rotations employed in the derivation of the angles $\gamma$ and $\chi$ in the line absorption terms $\phi_{I}, \phi_{Q}, \phi_{U}, \phi_{V}$. They give the transformation from the $\{x y z\}$ reference frame in which only $B_{z}$ is non-zero to the $\left\{x^{\prime \prime \prime} y^{\prime \prime \prime} z^{\prime \prime \prime}\right\}$ reference frame of the outgoing pencil of radiation (which points in the $z^{\prime \prime \prime}$-direction). In the latter frame the 3 components of $\boldsymbol{B}$ are in general non-zero. The angle $\gamma$ extends between $\boldsymbol{B}$ and $z^{\prime \prime \prime}$, the azimuth $\chi$ is given relative to the $x^{\prime \prime \prime}$-axis. For clarity's sake, both $\gamma$ and $\chi$ are not shown in the figure.

whence in Eqs. (5)-(11) $\gamma$ has to be replaced by $\pi-\gamma$ and $\chi$ by $\pi-\chi$. The $\phi_{I Q U V}(-\boldsymbol{n})$ and the $\phi_{I Q U V}^{\prime}(-\boldsymbol{n})$ can now be expressed in terms of the $\phi_{I Q U V}(+\boldsymbol{n})$ and the $\phi_{I Q U V}^{\prime}(+\boldsymbol{n})$ :

$$
\begin{aligned}
\phi_{I}(-\boldsymbol{n}) & =+\phi_{I}(+\boldsymbol{n}) \\
\phi_{Q}(-\boldsymbol{n}) & =+\phi_{Q}(+\boldsymbol{n}) \\
\phi_{U}(-\boldsymbol{n}) & =-\phi_{U}(+\boldsymbol{n}) \\
\phi_{V}(-\boldsymbol{n}) & =-\phi_{V}(+\boldsymbol{n})
\end{aligned}
$$


$\phi_{Q}^{\prime}(-n)=+\phi_{Q}^{\prime}(+n)$

$\phi_{U}^{\prime}(-\boldsymbol{n})=-\phi_{U}^{\prime}(+\boldsymbol{n})$

$\phi_{V}^{\prime}(-n)=-\phi_{V}^{\prime}(+n)$.

Having established these transformations, we can apply Feautrier's scheme to the polarised radiative transfer problem without any restrictions as to the line profile. We split the Stokes vector of radiation into 2 parts: the outgoing radiation $\boldsymbol{I}(+\boldsymbol{n})$ and its counterpart $\boldsymbol{I}(-\boldsymbol{n})$ propagating in the opposite (inward) direction. The polarised RTE Eq. (1) can be written separately for the outgoing and the incoming radiation:

$$
\begin{aligned}
& \frac{\mathrm{d}}{\mathrm{d} s} \boldsymbol{I}(+\boldsymbol{n})=-\boldsymbol{K}(+\boldsymbol{n}) \boldsymbol{I}(+\boldsymbol{n})+\boldsymbol{j}(+\boldsymbol{n}) \\
& -\frac{\mathrm{d}}{\mathrm{d} s} \boldsymbol{I}(-\boldsymbol{n})=-\boldsymbol{K}(-\boldsymbol{n}) \boldsymbol{I}(-\boldsymbol{n})+\boldsymbol{j}(-\boldsymbol{n})
\end{aligned}
$$

$\boldsymbol{K}(-\boldsymbol{n})$ and $\boldsymbol{j}(-\boldsymbol{n})$ have to be expressed in terms of $\boldsymbol{K}(+\boldsymbol{n})$ and $\boldsymbol{j}(+\boldsymbol{n})$ respectively. Using Eqs. (17)-(23) and introducing the modified Stokes vector $\tilde{\boldsymbol{I}}(-\boldsymbol{n})=\{I, Q,-U,-V\}^{\dagger}$ we can finally rewrite Eq. (25) as

$$
-\frac{\mathrm{d}}{\mathrm{d} s} \tilde{\boldsymbol{I}}(-\boldsymbol{n})=-\boldsymbol{K}(+\boldsymbol{n}) \tilde{\boldsymbol{I}}(-\boldsymbol{n})+\boldsymbol{j}(+\boldsymbol{n})
$$

With the definition of generalised Feautrier vectors

$$
\begin{aligned}
& \boldsymbol{J}=(\boldsymbol{I}(+\boldsymbol{n})+\tilde{\boldsymbol{I}}(-\boldsymbol{n})) / 2 \\
& \boldsymbol{H}=(\boldsymbol{I}(+\boldsymbol{n})-\tilde{\boldsymbol{I}}(-\boldsymbol{n})) / 2
\end{aligned}
$$

addition and subtraction of Eqs. (24) and (26) results in

$$
\begin{aligned}
\frac{\mathrm{d}}{\mathrm{d} s} \boldsymbol{J} & =-\boldsymbol{K} \boldsymbol{H} \\
\frac{\mathrm{d}}{\mathrm{d} s} \boldsymbol{H} & =-\boldsymbol{K} \boldsymbol{J}+\boldsymbol{j} .
\end{aligned}
$$

Finally, by elimination of $\boldsymbol{H}$ we arrive at the Feautrier-type second-order equation for $\boldsymbol{J}$

$\frac{\mathrm{d}}{\mathrm{d} s}\left(\boldsymbol{K}^{-1} \frac{\mathrm{d}}{\mathrm{d} s} \boldsymbol{J}\right)=\boldsymbol{K} \boldsymbol{J}-\boldsymbol{j}$

which is very similar to the classical non-magnetic solution for static atmospheres, valid for arbitrary blends. The choice between our polarised formal solver and the one developed by Rees et al. (1989) depends on the problem to be solved. In the presence of macroscopic velocity fields, one has to consider the outgoing Stokes vector $\boldsymbol{I}(+v,+\boldsymbol{n})=\{I, Q, U, V\}^{\dagger}$ on one side of the line, the incoming Stokes vector $\tilde{\boldsymbol{I}}(-v,-\boldsymbol{n})=$ $\{I, Q,-U, V\}^{\dagger}$ on the other side of the line; the line profile has to be symmetric, blends are not permitted. In a static atmosphere, blends can correctly be dealt with, placing outgoing Stokes vector $\boldsymbol{I}(+\boldsymbol{n})=\{I, Q, U, V\}^{\dagger}$ and incoming Stokes vector $\tilde{\boldsymbol{I}}(-\boldsymbol{n})=\{I, Q,-U,-V\}^{\dagger}$ on the same side of the line.

\section{Radiative accelerations in a magnetic atmosphere}

The radiative acceleration vector due to lines is given by

$\boldsymbol{g}_{i}^{\mathrm{rad}}=\sum_{k, m>k} f n_{i k} \int_{\Omega} \int_{v}(\boldsymbol{e} \cdot \boldsymbol{I}) \boldsymbol{\Omega} \mathrm{d} \Omega \mathrm{d} v$ where

- $f=1 /\left(n_{i} A m_{\mathrm{p}} c\right)$,

- with $n_{i}$ the total number density $\left(\mathrm{cm}^{-3}\right)$ of ions $A^{+i}, n_{i k}$ the number density of ions in initial lower level $k$,

- and the summation extending over all transitions from initial level $k$ to higher levels $m$.

- $A m_{\mathrm{p}}$ is the mass $(\mathrm{g})$ of the ion,

- $(\boldsymbol{e} \cdot \boldsymbol{I})$ denotes the inner product of the vector $\boldsymbol{e}=$ $\kappa_{\circ}\left\{\phi_{I}, \phi_{Q}, \phi_{U}, \phi_{V}\right\}$ with the Stokes vector $\boldsymbol{I}$.

Let us emphasise again that the angles $\gamma$ and $\chi$ in the vector $\boldsymbol{e}$ do not depend on the direction of the magnetic field vector relative to some hypothetical observer but relative to the direction of the pencil of radiation. The latter is characterised in our geometry by the angles $\theta$ relative to the vertical and $\psi$ about the vertical, starting from the plane defined by the magnetic field vector $\boldsymbol{B}$ and the vertical. $\boldsymbol{B}$ is inclined by the angle $\beta$ towards the vertical (see Fig. 1).

The respective $\phi_{I Q U V}$ for the outgoing and the incoming radiation are not identical (see Eqs. (17)-(23), one has duly to take this into account when calculating the integral (32)). We split the latter into 2 parts corresponding to the contributions from the outgoing and from the incoming radiation. Defining

$\boldsymbol{e}^{+}=\kappa_{\circ}\left\{\phi_{I}(+\boldsymbol{n}), \phi_{Q}(+\boldsymbol{n}), \phi_{U}(+\boldsymbol{n}), \phi_{V}(+\boldsymbol{n})\right\}$

$\boldsymbol{e}^{-}=\kappa_{\circ}\left\{\phi_{I}(-\boldsymbol{n}), \phi_{Q}(-\boldsymbol{n}), \phi_{U}(-\boldsymbol{n}), \phi_{V}(-\boldsymbol{n})\right\}$

the calculation of the integral now involves evaluation of the respective inner products $I(+\boldsymbol{n}) \cdot \boldsymbol{e}^{+}$and $I(-\boldsymbol{n}) \cdot \boldsymbol{e}^{-}$. Remembering that our Zeeman Feautrier method yields $\tilde{\boldsymbol{I}}(-\boldsymbol{n})=\{I, Q,-U,-V\}^{\dagger}$ for the incoming radiation and keeping in mind the changes in sign of the $\phi_{I Q U V}$ according to Eqs. (17)-(20), we obtain for the vertical and the horizontal radiative acceleration respectively

$g_{i, \mathrm{v}}^{\mathrm{rad}}=\sum_{k, m>k} n_{i k} \int_{0}^{1} \int_{0}^{2 \pi} \int_{0}^{\infty} F^{+} \mu \mathrm{d} \mu \mathrm{d} \psi \mathrm{d} v$

$g_{i, \mathrm{~h}}^{\mathrm{rad}}=\sum_{k, m>k} n_{i k} \int_{0}^{1} \int_{0}^{2 \pi} \int_{0}^{\infty} F^{+} \sqrt{1-\mu^{2}} \mathrm{~d} \mu \cos \psi \mathrm{d} \psi \mathrm{d} \nu$

where $F^{+}=2 f\left(\boldsymbol{H} \cdot \boldsymbol{e}^{+}\right)$. This means that whereas in order to obtain the mean intensity $\boldsymbol{I}(+\boldsymbol{n})+\boldsymbol{I}(-\boldsymbol{n})$ (required for example in the determination of ionisation rates) one has to replace the Stokes parameters $U$ and $V$ in $\tilde{\boldsymbol{I}}(-\boldsymbol{n})$ by $-U$ and $-V$ respectively, no such replacements are necessary for the calculation of the acceleration integral (35) since the changes in sign of the Stokes $U$ and $V$ parameters are accompanied by changes in $\phi_{U}$ and $\phi_{V}$ respectively.

\section{Revised amplifications of the radiative accelerations due to Zeeman splitting}

As long as the magnetic field strength is zero, expression (35) with our new Zeeman Feautrier method and the correct inner product $(\boldsymbol{e} \cdot \boldsymbol{I})$ gives radiative accelerations that are of course identical to those given in Paper I; they are therefore not shown again. For non-zero magnetic fields however, all the amplifications due to Zeeman splitting presented and analysed in Paper I 
should be revised. This section is then devoted to this revision and to a discussion of the new amplifications.

\subsection{Amplifications and field strength}

The new amplifications are shown in Fig. 2 and have been computed with the same model atmosphere (a Kurucz Atlas9 model with $T_{\text {eff }}=12000 \mathrm{~K}$ and $\left.\log g=4.0\right)$ and the same atomic database (VALD, see Piskunov et al. 1995) as in Paper I. Amplifications are based on the simplified expression for the total radiative accelerations $g^{\text {rad }}$ given by Eq. (17) of Paper I. The magnetic field is vertical. The most striking difference compared to Fig. 5 of Paper I is that the amplifications $\left(\varepsilon=\log g^{\mathrm{rad}}\left(\right.\right.$ magnetic) $-\log g^{\mathrm{rad}}$ (nonmagnetic)) remain smaller than about 0.22 and 0.40 dex respectively for $1 \mathrm{~T}$ and $4 \mathrm{~T}$. This corresponds to a maximum increase of radiative accelerations by a factor 2.5 . This increase is by no means negligible, but it is also far from the huge amplifications found for a few metals in Paper I. The Ag acceleration decreases strongly as shown in Fig. 3 and this case will be discussed in more detail in Sect. 4.3. The elements most sensitive to Zeeman desaturation and ensuing amplification at $4 \mathrm{~T}(\varepsilon$ larger than $0.2 \mathrm{dex})$ appear to be: $\mathrm{Mg}, \mathrm{Zn}, \mathrm{Ca}, \mathrm{Hg}, \mathrm{Fe}, \mathrm{Ni}, \mathrm{S}, \mathrm{Mn}, \mathrm{Ga}, \mathrm{P}, \mathrm{Ge}, \mathrm{Co}$. The extrema corresponding to the largest $|\varepsilon|$ are listed in Table 1. In most cases, as one can see in Fig. 2, they correspond to amplifications (positive extrema of $\varepsilon$ ). Elements for which the radiative acceleration decreases due to Zeeman splitting (negative extrema of $\varepsilon$ ) are listed at the bottom of Table 1 (in the columns labeled "El." they are marked by "(-)"). Our new calculations show that, contrary to what we found in Paper I, there are a few elements in addition to $\mathrm{Ag}$ which experience such a decrease.

\subsection{Amplifications and field angle}

We have re-explored the effect of field angle on the accelerations. At variance with what has been affirmed in Paper I, our new computations do not show a strong dependence of radiative accelerations on field angle. When the angle of the magnetic field with respect to the vertical is increased from $0^{\circ}$ (vertical field) to $90^{\circ}$ (horizontal field), there is at most a $10 \%$ increase in amplification. This is in agreement with the estimation made by BM. We have found no instance of a maximum at an intermediate angle; only at field strengths of about $1 \mathrm{~T}$ simple triplets may display inverse behaviour (i.e. decrease when going from $0^{\circ}$ to $90^{\circ}$ ).

\subsection{The effects of strong blending}

The strong decrease (Fig. 3) in radiative acceleration of Ag for large magnetic fields $(2 \mathrm{~T}-4 \mathrm{~T})$, reveals that Zeeman splitting of lines does not invariably lead to an increase in radiative acceleration. This can be understood if we suppose that some of the relatively weak $\mathrm{Ag}$ lines overlap with strong lines of other elements. Zeeman splitting would lead to only a small increase in the cross section of $\mathrm{Ag}$ but to a very large drop in flux near the strong line. Once all Zeeman components of the $\mathrm{Ag}$ line are completely blended with the strong line there should be no further decrease in the acceleration with increasing field strength. This idea is compatible with the fact that there is little change in acceleration between $2 \mathrm{~T}$ and $4 \mathrm{~T}$ according to Fig. 3 .

In order to verify this scenario, we have computed radiative accelerations using exclusively the lines of $\mathrm{Ag}$. The results are presented at the bottom of Fig. 4 and clearly confirm our hypothesis: (i) comparing the solid lines (heavy - light), the radiative accelerations of $\mathrm{Ag}$ appear to be highly sensitive to the presence of other elements; (ii) comparing heavy curves to light ones for both $0 \mathrm{~T}$ and $4 \mathrm{~T}$, the decrease is found to occur only when all elements are present in the spectrum; (iii) comparing light lines (solid - dashed), the accelerations clearly are amplified, even if only slightly, by Zeeman splitting, the expected "normal" behaviour. In this latter case the small amplification values are the logical consequence of the fact that Ag lines are rather weak and only moderately saturated.

In comparison, we present in the same Fig. 4 the effects of blending on two other elements, viz. $\mathrm{Cr}$ and $\mathrm{Fe}$. Both elements react quite sensitively to blends because obviously the available flux is much reduced. However, the increase in cross section due to Zeeman splitting induced desaturation exceeds the decrease in radiative flux due to magnetically increased line blanketing, leading to an overall amplification.

To better understand what exactly happens in the case of $\mathrm{Ag}$, we have made detailed computations for that element. It emerged that the radiative acceleration of $\mathrm{Ag}$ is essentially due to the absorption through the Ag II resonance line $\lambda 1112.483 \AA$. Figure 5 shows the fluxes around this line in detail, both for $0 \mathrm{~T}$ (solid lines) and $4 \mathrm{~T}$ (dashed lines). We have considered 2 cases: selecting only the $\mathrm{Ag}$ lines from the atomic data file (upper spectra), and on the other hand taking all the lines from the VALD database. It is apparent that the Ag II resonance line is blended with the Fe II line at $\lambda 1112.465 \AA$ and that some other (weaker) lines are also involved (see Fig. 5). For the sake of clarity we will only consider the Fe II line in the following discussion. Without a magnetic field, the Ag II resonance line is slightly to the red of the core of the highly saturated Fe II line. In the presence of Zeeman splitting due to a strong magnetic field, the width of the Fe II line increases significantly: 2 of the 3 Zeeman components of the Ag II resonance line are now completely within the enlarged Fe II line core, reducing notably the photon flux in the Zeeman components of the Ag II line.

Here we want to make a few cautionary remarks. Ag does not follow the general behaviour of radiative accelerations which increase with magnetic field strength, because the very few lines from the VALD database which provide noticeable cross sections are all blended with stronger lines. This seems to be an exceptional case. Whether or not the behaviour of $\mathrm{Ag}$ would change with a more complete atomic line list can only be a matter of speculation. As far as the present VALD data are concerned, our result is especially sensitive to the accuracy of the wavelengths in the database as illustrated by Fig. 6 which demonstrates the effects of a line shift of $\Delta \lambda= \pm 5 \mathrm{~m} \AA$. Finally, the solar abundance of $\mathrm{Ag}$ is so small that even its resonance lines are not saturated. Therefore, the radiative acceleration of Ag does not significantly benefit from any desaturation 

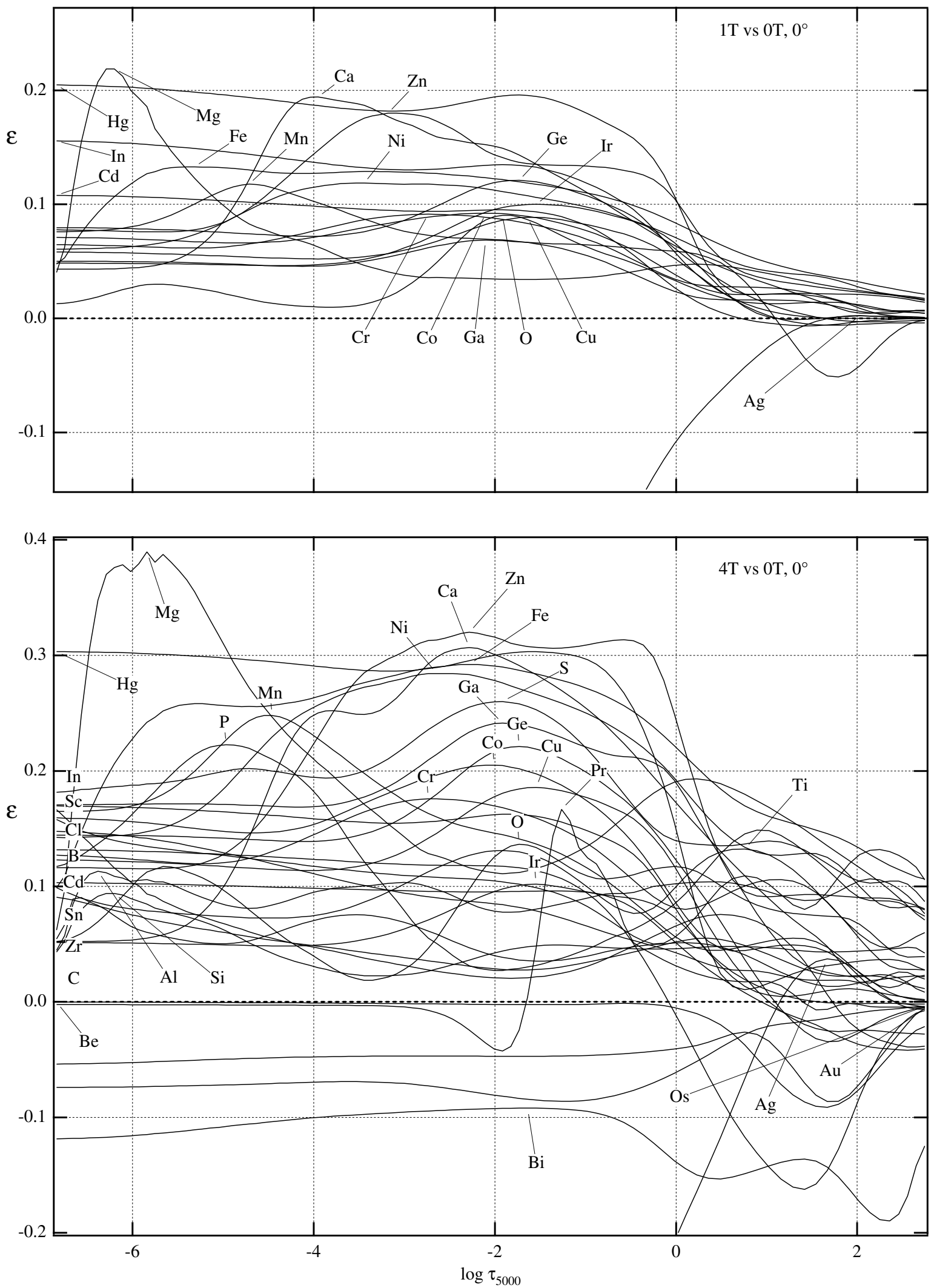

Fig. 2. Amplification of the radiative accelerations of the chemical elements due to Zeeman splitting as a function of optical depth and of magnetic field strength. Logarithmic amplifications $\varepsilon=\log g^{\text {rad }}$ (magnetic) $-\log g^{\text {rad }}$ (nonmagnetic) (the "nonmagnetic" accelerations are the same as those of Fig. 5 of Paper I), are displayed for field strengths of 1 and $4 \mathrm{~T}$ at a constant $0^{\circ}$ angle between magnetic field vector and vertical. Curves are plotted only when $|\varepsilon|>0.0413927$ dex (10\% amplification) at any depth point. The tag for each element points to the location of maximum value. In the figure for $4 \mathrm{~T}$, the crowded left part pertains to the following elements: $\mathrm{B}, \mathrm{C}, \mathrm{Cl}, \mathrm{Sc}, \mathrm{Zr}, \mathrm{Cd}, \mathrm{In}, \mathrm{Sn}$. Parts of the curves for $\mathrm{Ag}$ are cut off in these figures, but the case of $\mathrm{Ag}$ is discussed in detail in Sect. 4.3 (see also Fig. 3). 
Table 1. Extrema of $\varepsilon$ for field strengths of 1,2 , and $4 \mathrm{~T}$ at a constant $0^{\circ}$ angle between magnetic field vector and vertical. For each field strength, elements are sorted according to the extrema of $\varepsilon$ and the column labeled $\log \tau$ gives the optical depth (at $5000 \AA$ ) of the corresponding layer. The elements listed are those selected for Fig. 2. Elements for which the extreme value is negative are marked by "(-)".

\begin{tabular}{|c|c|c|c|c|c|c|c|c|}
\hline \multicolumn{3}{|c|}{$1 \mathrm{~T}$} & \multicolumn{3}{|c|}{$2 \mathrm{~T}$} & \multicolumn{3}{|c|}{$4 \mathrm{~T}$} \\
\hline El. & extr. $\varepsilon$ & $\operatorname{lng} \tau$ & El. & extr. $\varepsilon$ & $\operatorname{lng} \tau$ & El. & extr. $\varepsilon$ & $\operatorname{lng} \tau$ \\
\hline $\mathrm{Mg}$ & 0.219 & -6.20 & $\mathrm{Mg}$ & 0.334 & -6.11 & $\mathrm{Mg}$ & 0.390 & -5.84 \\
\hline $\mathrm{Hg}$ & 0.205 & -6.84 & $\mathrm{Hg}$ & 0.329 & -6.84 & $7 n$ & 0.320 & -2.28 \\
\hline $\mathrm{Ca}$ & 0.194 & -3.95 & $\mathrm{Zn}$ & 0.282 & -2.73 & $\mathrm{Ca}$ & 0.307 & -2.28 \\
\hline $7 n$ & 0.180 & -3.18 & $\mathrm{Ca}$ & 0.260 & -2.82 & $\mathrm{Hg}$ & 0.303 & -6.84 \\
\hline In & 0.156 & -6.84 & $\mathrm{Fe}$ & 0.210 & -2.46 & $\mathrm{Fe}$ & 0.292 & -2.28 \\
\hline $\mathrm{Fe}$ & 0.133 & -5.31 & $\mathrm{Ni}$ & 0.199 & -2.73 & $\mathrm{Ni}$ & 0.284 & -2.64 \\
\hline $\mathrm{Ge}$ & 0.121 & -1.73 & $\mathrm{Ge}$ & 0.192 & -1.73 & $S$ & 0.260 & -1.92 \\
\hline $\mathrm{Ni}$ & 0.119 & -3.44 & $\mathrm{Mn}$ & 0.185 & -4.63 & $\mathrm{Mn}$ & 0.248 & -4.46 \\
\hline $\mathrm{Mn}$ & 0.118 & -4.71 & In & 0.160 & -6.84 & Ga & 0.241 & -1.92 \\
\hline $\mathrm{Cd}$ & 0.108 & -6.84 & $\mathrm{~S}$ & 0.156 & -2.01 & $\mathrm{P}$ & 0.223 & -4.97 \\
\hline $\mathrm{Ir}$ & 0.100 & -1.55 & $\mathrm{Ga}$ & 0.153 & -1.64 & Ge & 0.221 & -1.73 \\
\hline Co & 0.092 & -2.10 & $\mathrm{P}$ & 0.148 & -5.14 & Co & 0.205 & -2.01 \\
\hline $\mathrm{Cr}$ & 0.091 & -2.73 & Co & 0.148 & -2.01 & $\mathrm{Cu}$ & 0.186 & -1.55 \\
\hline $\mathrm{O}$ & 0.090 & -1.92 & $\mathrm{Cu}$ & 0.135 & -1.55 & $\mathrm{Cr}$ & 0.176 & -2.73 \\
\hline $\mathrm{Cu}$ & 0.089 & -1.64 & $\mathrm{Cr}$ & 0.133 & -2.73 & In & 0.170 & -6.84 \\
\hline $\mathrm{Ga}$ & 0.069 & -2.10 & $\mathrm{Cd}$ & 0.126 & -6.84 & $\mathrm{Pr}$ & 0.167 & -1.26 \\
\hline $\operatorname{Ag}(-)$ & -0.425 & -6.84 & $\mathrm{O}$ & 0.121 & -1.82 & Sc & 0.166 & -6.84 \\
\hline & & & Sc & 0.119 & -6.84 & $\mathrm{Cl}$ & 0.158 & -6.84 \\
\hline & & & $\mathrm{Ti}$ & 0.101 & -1.92 & B & 0.148 & -6.84 \\
\hline & & & $\mathrm{A} 1$ & 0.093 & -6.38 & $\mathrm{O}$ & 0.136 & -1.73 \\
\hline & & & $\mathrm{Ir}$ & 0.093 & -1.64 & $\mathrm{Ti}$ & 0.132 & 0.76 \\
\hline & & & $\mathrm{Cl}$ & 0.089 & -6.84 & $\mathrm{~A} 1$ & 0.113 & -6.38 \\
\hline & & & B & 0.086 & -6.84 & $\mathrm{~N}$ & 0.106 & -5.23 \\
\hline & & & $\mathrm{Y}(-)$ & -0.088 & -6.84 & Sn & 0.103 & -6.84 \\
\hline & & & $\mathrm{Bi}(-)$ & -0.155 & 2.25 & $\mathrm{Ir}$ & 0.102 & -1.55 \\
\hline & & & $\mathrm{Ag}(-)$ & -0.607 & -6.84 & $\mathrm{Si}$ & 0.094 & -6.20 \\
\hline & & & & & & $\mathrm{Zr}$ & 0.091 & -6.84 \\
\hline & & & & & & C & 0.090 & -6.56 \\
\hline & & & & & & Os(-) & -0.086 & -1.17 \\
\hline & & & & & & $\mathrm{Au}(-)$ & -0.086 & 1.67 \\
\hline & & & & & & $\mathrm{Be}(-)$ & -0.091 & 1.67 \\
\hline & & & & & & $\mathrm{Cd}(-)$ & -0.162 & 1.42 \\
\hline & & & & & & $\operatorname{Bi}(-)$ & -0.190 & 2.36 \\
\hline & & & & & & $\mathrm{Ag}(-)$ & -0.564 & -6.84 \\
\hline
\end{tabular}

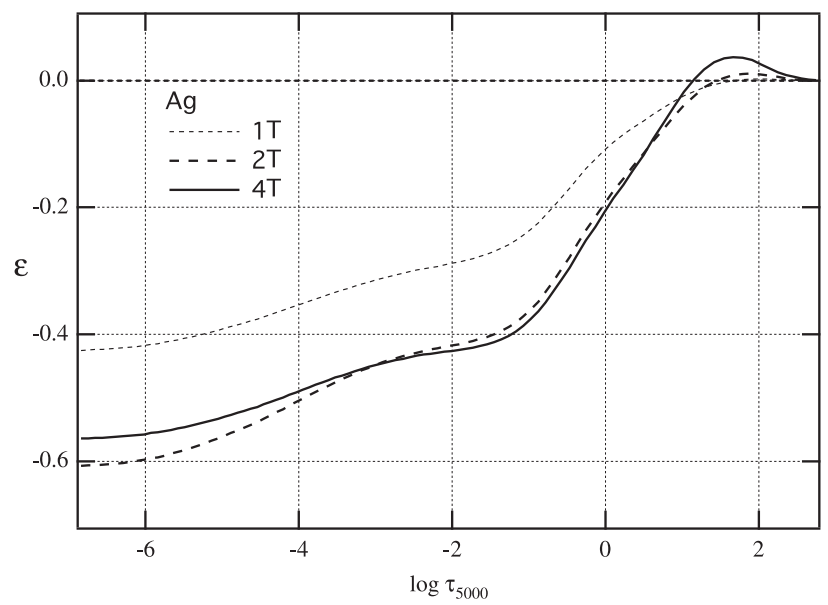

Fig. 3. Effect of Zeeman splitting on Ag. This plot displays results only for Ag; results for the other elements are shown in Fig. 2. The acceleration strongly decreases when the magnetic field intensity goes from 0 to $2 \mathrm{~T}$, but there is no further decrease towards $4 \mathrm{~T}$. This is due to severe blending as detailed in the text.

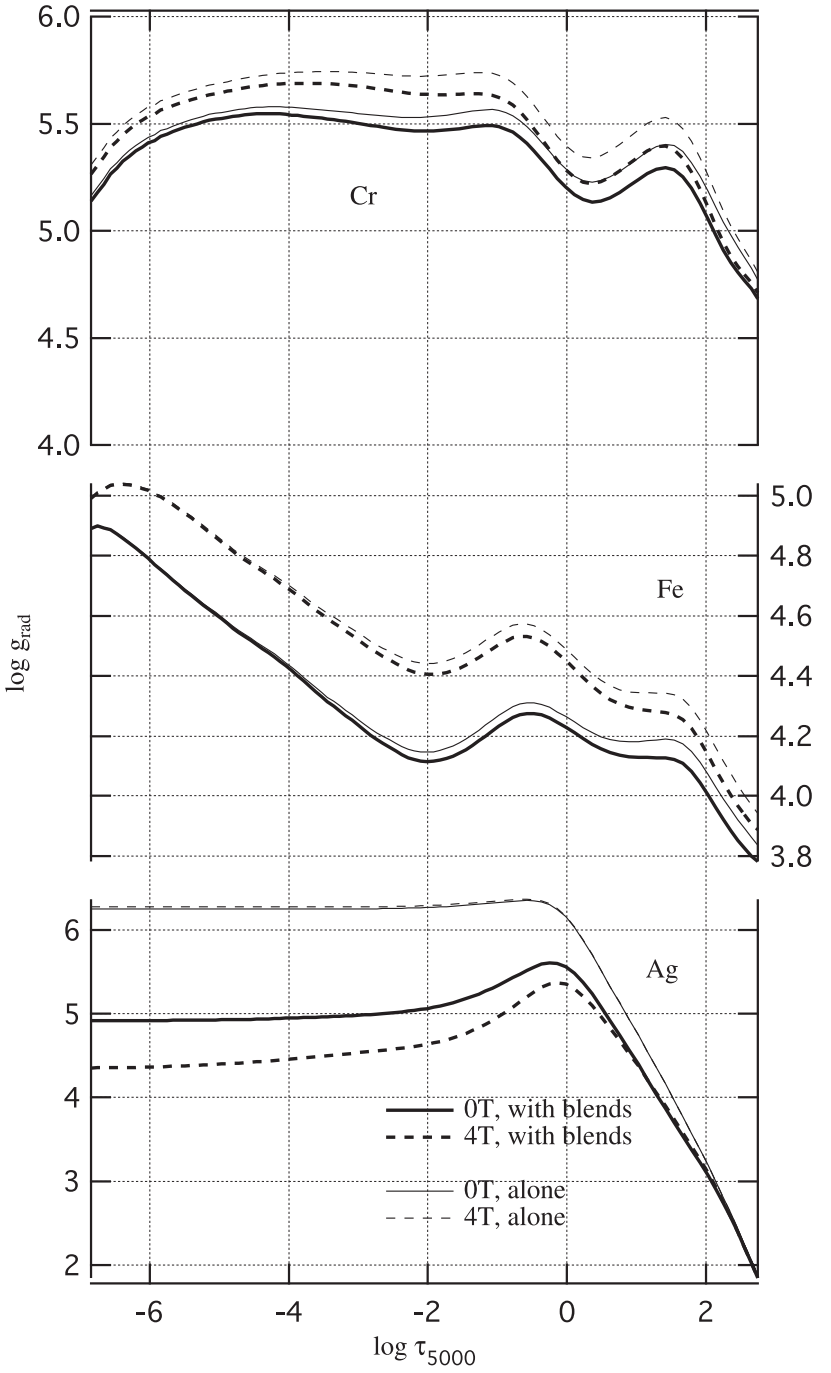

Fig. 4. Effect of blends on $\mathrm{Cr}$, Fe, and $\mathrm{Ag}$ in the $12000 \mathrm{~K}$ model.

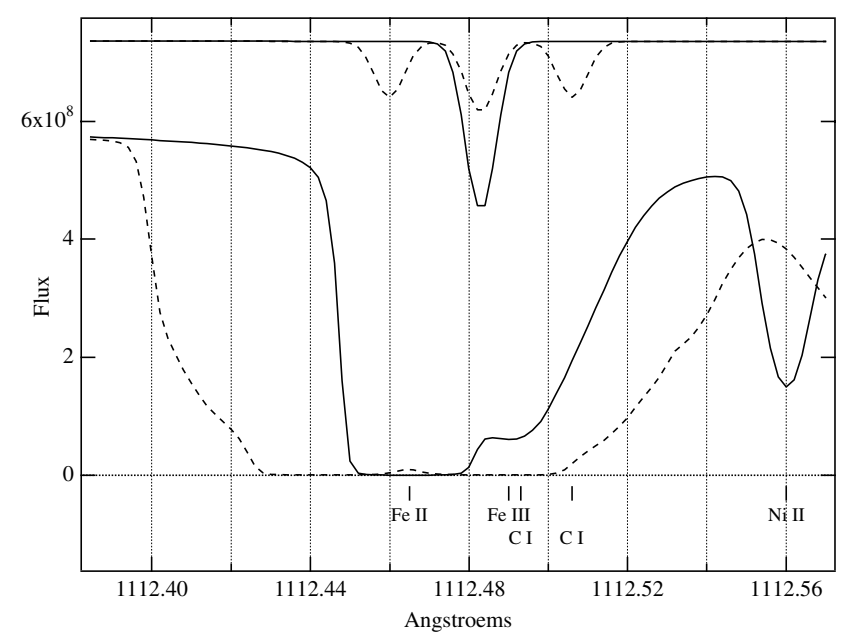

Fig. 5. Flux at $\log \tau_{5000}=-6.020$ around the Ag II resonance line $\lambda 1112.483 \AA$ A. Solid lines correspond to the zero field case, dashed lines correspond to a $4 \mathrm{~T}$ field. The upper spectra are based on Ag lines only. The spectra at the bottom are based on all lines in the VALD database. The Ag line is mainly blended with the Fe II line $\lambda 1112.465 \AA$, and to a lesser extent with Fe III $\lambda 1112.490 \AA$, C I $\lambda 1112.493 \AA$, and C I $\lambda 1112.506 \AA$. 


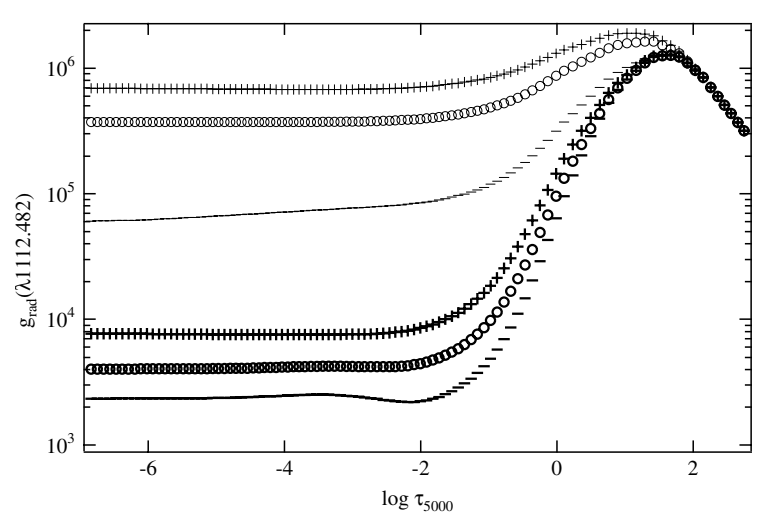

Fig. 6. Radiative acceleration of $\mathrm{Ag}$ II due to the resonance line $\lambda 1112.483 \AA$. The curves with "+" markers correspond to a wavelength shift of $+5 \mathrm{~m} \AA$, “-” for $-5 \mathrm{~m} \AA$, "o" no shift. Heavy markers pertain to $4 \mathrm{~T}$.

through Zeeman splitting, which makes Ag especially sensitive to blending.

In this section we have so far discussed the case that Zeeman splitting leads to a weak line being increasingly "embedded" in a strong line. One could imagine a similar situation but with the strong line being rather insensitive to Zeeman splitting and the weak line displaying a very large splitting. We would not exclude the possibility of amplification but a systematic exploration of these scenarios is outside the scope of our paper. Note that so far we have assumed solar abundances for all elements and that enhanced blending in CP stars can lead to quite different results from those presented here. And finally we must not forget that chemical stratification in the CP star atmospheres add more parameters to the problem of magnetically enhanced radiative accelerations.

\subsection{Changing the effective temperature}

We have also made computations for models with other effective temperature $(10000 \mathrm{~K}$ and $14000 \mathrm{~K})$ to see whether new trends would emerge. As expected, zero field radiative accelerations change significantly from one model to the other. This is to be expected since the relative populations of ions are clearly different at a given optical depth when the effective temperature is changed by $\pm 2000 \mathrm{~K}$. On the other hand, our atomic database is rather incomplete for higher ionisation stages which makes the present computations somewhat unreliable for $14000 \mathrm{~K}$ and detailed comparison between the models thus irrelevant. Nevertheless, we have observed that globally, amplifications due to Zeeman splitting are of the same order of magnitude for $14000 \mathrm{~K}$ as for $12000 \mathrm{~K}$ or $10000 \mathrm{~K}$. A closer look reveals that strong variations of the accelerations with effective temperature cannot simply be attributed to the lack of atomic data as mentioned above. For instance, if some metals are less sensitive to Zeeman splitting at lower temperatures while others are more sensitive and still other elements display almost invariant behaviour, this is certainly related to the dependence of the relative intensities of the spectral lines on effective temperature. We have found it impossible to establish any systematic trends.

\subsection{Zeeman patterns and amplifications}

In the light of Fig. 2, it would seem that the claim by BM that even in the most favourable case (complex Zeeman pattern and horizontal field), the amplification factor of individual lines is limited to a value of 2.3 is justified after all, contrary to what we have claimed in Paper I. One has however to be cautious: our results reflect amplifications due to thousands of sometimes heavily blended lines in a fairly realistic Kurucz atmosphere, whereas the statement by BM is valid only for the Unno (1956) approximation in a Milne-Eddington atmosphere, excluding anomalous dispersion effects. In their numerical solutions, BM never considered field strengths exceeding $1.5 \mathrm{~T}$. It is thus in no way surprising that they never found amplification factors in excess of 2.

A direct comparison for the Sr II $\lambda 4077$ line reveals (Fig. 7) reasonable agreement between the results of BM and our calculations, at least as long as magneto-optical effects are not taken into account: the latter can have dramatic repercussions on the horizontal accelerations whose sign can now change with optical depth, although they still remain fairly small. Amplifications of individual lines with very complex Zeeman patterns however can become much larger than 0.4 dex once the Unno solution is dropped for the Zeeman Feautrier solution. Extensive calculations for the 20 Zeeman patterns listed in Table 1 of Stift \& Leone (2003) reveal that this is indeed the case; in a very strong ( $930 \mathrm{~m} \AA$ zero field equivalent width) fictitious Fe II line situated at $4500 \AA ̊$ one encounters amplifications of $0.8 \mathrm{dex}$ at $2 \mathrm{~T}$ and more than $1.1 \mathrm{dex}$ at $6 \mathrm{~T}$ (Fig. 8). Note that these values are not weighted by the actual population of Fe II whose fraction exceeds 0.85 in the outer layers.

The effects of anomalous dispersion are not only important for the horizontal accelerations. They turn out to be by no means negligible in the determination of vertical accelerations. The omission of magneto-optical terms in the line absorption matrix would lead to an underestimation of accelerations by up to 0.14 dex in a $2 \mathrm{~T}$ vertical magnetic field, by up to 0.20 dex in a $6 \mathrm{~T}$ horizontal field (please note the opposite sign of this effect as compared to Paper I). In both cases we find a very strong dependence on the Zeeman pattern (Fig. 9).

The relevance of these results lies in the fact that for many chemical elements line lists are still incomplete and that the situation concerning Landé factors is far from satisfactory. VALD data for twice ionised rare earths for example are almost completely lacking; $\mathrm{Ce}, \mathrm{Nd}, \mathrm{Eu}$ and $\mathrm{Er}$ are the only exceptions. If the Zeeman patterns of the rare earths are more complex on the average than the Zeeman patterns of $\mathrm{Mg}, \mathrm{Zn}, \mathrm{Ca}, \mathrm{Hg}$ and $\mathrm{Fe}$ (the elements which display the largest amplifications in our study) their true amplifications could conceivably exceed the present limit of $0.4 \mathrm{dex}$ in $\mathrm{CP}$ star atmospheres where the rare earths are found to be overabundant. Taking the VALD data, we note that each line of Pr II and of Nd II is split on average into 34 Zeeman components, each line of Tb II and of Dy II into 40 components. This has to be compared to 12 and 18 components respectively for $\mathrm{Ca}$ II and $\mathrm{Fe}$ II. 

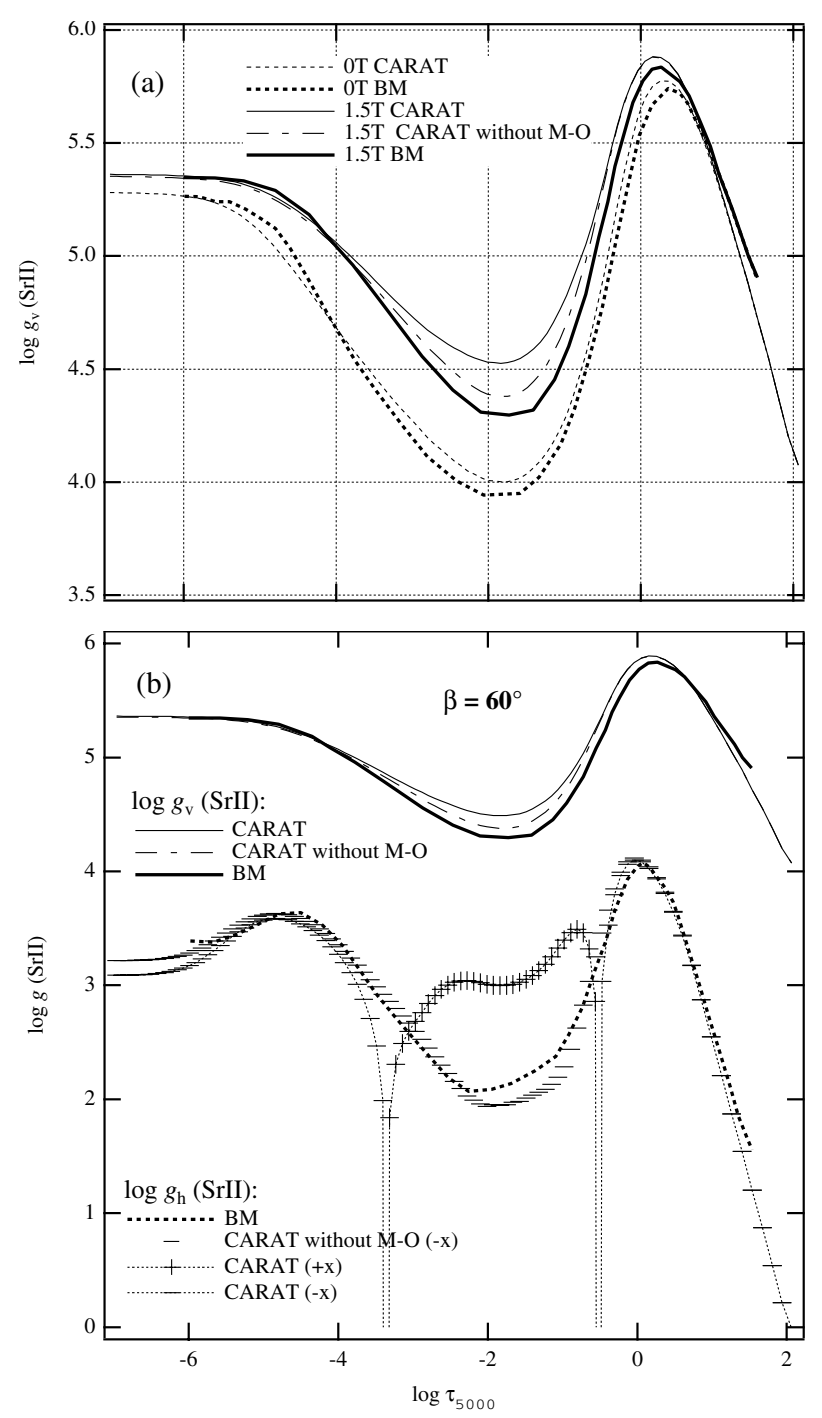

Fig. 7. Radiative accelerations due to the $\operatorname{Sr}$ II $\lambda 4077$ line in the nonmagnetic case and in a $1.5 \mathrm{~T}$ field, based on a $8500 \mathrm{~K}, \log g=4.0$ Kurucz (1993) model atmosphere. Top panel a): comparison between the vertical accelerations obtained by BM and our results, both with and without magneto-optical effects. The field is vertical. Bottom panel b): similar to top panel, but with vertical (top) and horizontal (bottom) accelerations. The field is inclined by $60^{\circ}$ with respect to the vertical. Note that the horizontal acceleration changes sign twice over optical depth when M.-O. effects are taken into account.

\section{Conclusions}

We have revisited the formula for the radiative acceleration vector in the presence of magnetic fields (Eq. (12) of Paper I). The realisation that the Zeeman Feautrier method as presented by Rees et al. (1989) is only valid for symmetric line profiles in conjunction with the fact that those line absorption terms which change sign for the incoming radiation had been incorrectly identified, has led us to establish a new Zeeman Feautrier formulation valid for (heavily) blended lines in static atmospheres and to derive the correct signs of the absorption coefficients entering Eq. (12) of Paper I.

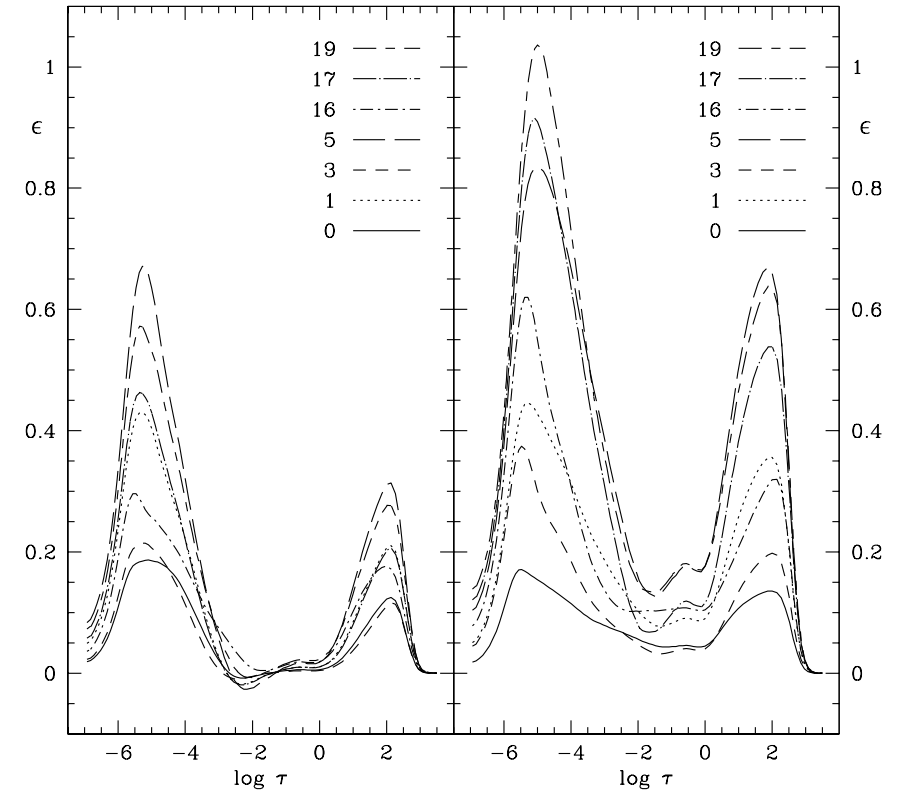

Fig. 8. Amplification $\varepsilon$ of radiative accelerations as a function of Zeeman pattern and of magnetic field strength. The atomic parameters of a strong (fictitious) Fe II line at $4500 \AA$ are chosen such that the zero field equivalent width is $930 \mathrm{~m} \AA$ in a $12000 \mathrm{~K}, \log g=4.0$ Kurucz (1993) model atmosphere. Accelerations are not weighted by the population of Fe II. The left panel pertains to a $2 \mathrm{~T}$, the right panel to a $6 \mathrm{~T}$ horizontal magnetic field. The labels of the various curves correspond to the pattern numbers given in Table 1 of Stift \& Leone (2003).

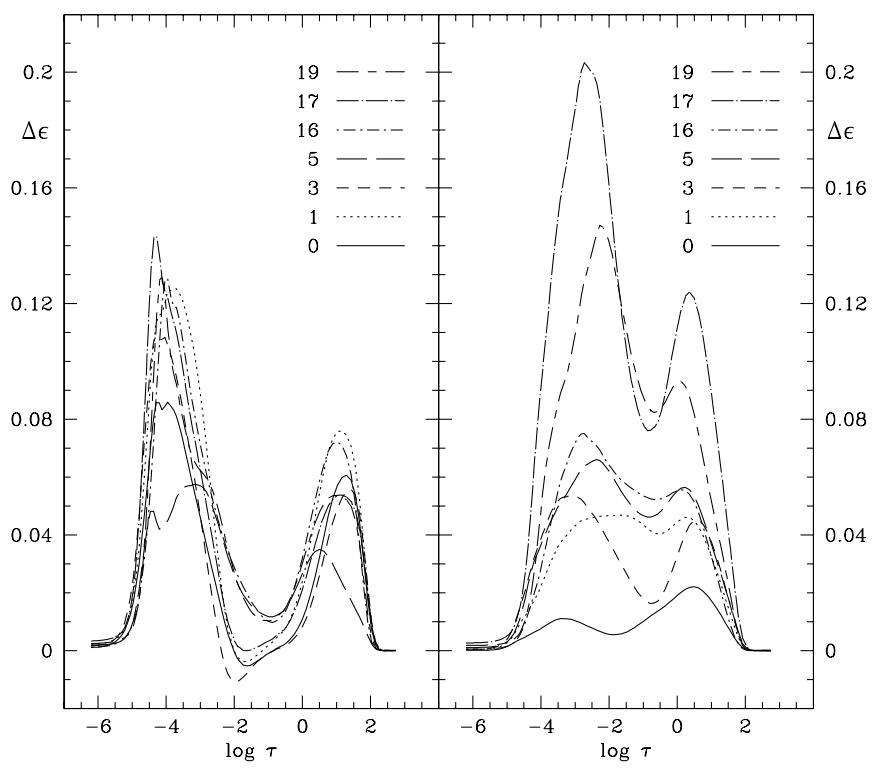

Fig. 9. Difference in amplification $\Delta \varepsilon=\varepsilon_{\text {with mo }}-\varepsilon_{\text {without mo }}$ of radiative accelerations, calculated for the same fictitious Fe II line as in Fig. 8 with anomalous dispersion terms included and without, as a function of Zeeman pattern and of magnetic field strength. The left panel pertains to a $2 \mathrm{~T}$ vertical, the right panel to a $6 \mathrm{~T}$ horizontal magnetic field; labels have the same meaning as in Fig. 8.

A thorough reevaluation of the results and conclusions presented in Paper I thus became necessary. In addition, we wanted to explore just how important blends are in the calculation of 
radiative accelerations and how the different chemical elements react to blending. As it turns out, the amplifications of several elements are now drastically reduced (at most 0.40 dex at $4 \mathrm{~T}$ ) as compared to the claims made in Paper I; all other elements exhibit some modest reduction. The strong angle effect also disappears. Thus we can no longer anticipate the emergence, as a consequence of angle-sensitive magnetic amplification, of complex horizontal structures related to the field topology. Still, one has to recall that the high dependency of the diffusion velocities on the inclination of the magnetic field lines should lead to horizontal abundance inhomogeneities.

But our new results show that amplifications still remain far from negligible for many chemical elements, in particular $\mathrm{Mg}, \mathrm{Zn}, \mathrm{Ca}, \mathrm{Hg}, \mathrm{Fe}, \mathrm{Ni}, \mathrm{S}, \mathrm{Mn}, \mathrm{Ga}, \mathrm{P}, \mathrm{Ge}, \mathrm{Co}$. Amplification factors of up to 2.5 can be found at solar abundances and $4 \mathrm{~T}$ field strengths. Magneto-optical effects continue to play a role, so the anomalous dispersion terms in the line absorption matrix must not be omitted. Amplifications in lines with complex Zeeman patterns may exceed $1 \mathrm{dex}$ and it is likely that with more complete atomic data available, some rare earths may exhibit really large amplifications. This is no mere speculation: calculations for Eu reveal that amplifications in a vertical $4 \mathrm{~T}$ field and at 100 times the solar abundance - not unusual for a CP star - exceed those of Fe, although our list contains just $152 \mathrm{Eu}$ II and $4 \mathrm{Eu}$ III lines.

When blending is taken into account, radiative accelerations are significantly lowered compared to results that are based only on the lines of a single element. The effect can range from about 0.2 dex to more than 1 dex in a few cases (see Fig. 4). In blended spectra, most elements still exhibit enhanced accelerations in the presence of Zeeman splitting but we have found a small number of elements with few and weak lines in the atomic databases blends which behave the opposite way: Zeeman splitting actually reduces accelerations instead of enhancing them.
This leaves us with the conclusion that despite the downward revision of the accelerations given in Paper I there can be little doubt that the diffusion of chemical elements in CP star atmospheres is seriously affected by Zeeman splitting.

Acknowledgements. Thanks go to Georges Michaud for extremely useful discussions and to Egidio Landi Degl'Innocenti for his continuous help in understanding some of the many subtleties of magnetic polarisation. We are grateful to the referee, Marco Landolfi, for constructive criticism which has led to significant improvements in the presentation. MJS acknowledges support by the Austrian Science Fund $(F W F)$, project P16003-N05 "Radiation driven diffusion in magnetic stellar atmospheres" and a Visiting Professorship at the Observatoire de Paris-Meudon and Université Paris 7. Many of the calculations have been carried out on the Sgi Origin 3800 of the CINES in Montpellier.

\section{References}

Alecian, G., \& Stift, M. J. 2002, A\&A, 387, 271 (Paper I)

Auer, L. H., Heasley, J. N., \& House, L. L. 1977, ApJ, 216, 531

Babel, J., \& Michaud, G. 1991, A\&A, 241, 493 (BM)

Feautrier, P. 1964, CR Acad. Sci., Paris, 258, 3189

Kurucz, R. 1993, CD-ROM Model Distribution, Smithsonian Astrophys. Obs.

Mihalas, D. 1978, Stellar atmospheres, 2nd edition (W. H. Freeman, San Francisco)

Piskunov, N. E., Kupka, F., Ryabchikova, T. A., Weiss, W. W., \& Jeffery, C. S. 1995, A\&AS, 112, 525

Rees, D. E., \& Murphy, G. A. 1987, in Numerical Radiative Transfer, ed. W. Kalkofen (Cambridge: Cambridge Univ. Press), 241

Rees, D. E., Murphy, G. A., \& Durrant, C. J. 1989, ApJ, 339, 1093

Stift, M. J., \& Leone, F. 2003, A\&A, 398, 411

Unno, W. 1956, Publ. Astron. Soc. Jpn, 8, 108 